Service social

\title{
La consolidation et le développement des organismes communautaires : un élément de solution à la pauvreté
}

\section{Jean-Pierre Bélanger et Gilles Desrosiers}

Volume 44, numéro 3, 1995

Pauvreté

URI : https://id.erudit.org/iderudit/706710ar

DOI : https://doi.org/10.7202/706710ar

Aller au sommaire du numéro

Éditeur(s)

École de service social de l'Université Laval

ISSN

1708-1734 (numérique)

Découvrir la revue

Citer cet article

Bélanger, J.-P. \& Desrosiers, G. (1995). La consolidation et le développement des organismes communautaires : un élément de solution à la pauvreté. Service social, 44(3), 145-164. https://doi.org/10.7202/706710ar
Résumé de l'article

Dans un contexte où la pauvreté guette une partie croissante de la population, où les taux élevés de chômage et de dépendance à l'aide sociale stagnent et où l'État se retire graduellement de certains secteurs de la santé et des services sociaux, les organismes communautaires sont appelés à jouer un rôle davantage prédominant au sein de la société. Le présent article propose ainsi des moyens de consolider la présence et de favoriser le développement des organismes communautaires. À cet égard, il est posé que la création d'un fonds de développement communautaire, en plus d'assurer la survie économique des organismes, constitue un élément de solution à la pauvreté en contribuant à créer des emplois pour des personnes habituellement exclues du marché du travail, tout en permettant de répondre à des besoins essentiels pour la qualité de vie des personnes. 


\section{COMMENTAIRES ET DOCUMENTS}

\section{La consolidation et le développement des organismes communautaires: un élément de solution à la pauvreté}

Jean-Pierre BÉLANGER

Conseiller au Bureau du sous-ministre de la Santé et des Services sociaux

Gilles DESROSIERS

Professionnel de recherche,

Laboratoire de recherche en service social École de service social, Université Laval

\section{Remarque préliminaire}

Le texte qui suit aborde une question d'actualité, à savoir le financement et le rôle des organismes communautaires dans un contexte de pauvreté croissante et de désengagement de l'État. Fondé sur des idées originales, il illustre éloquemment l'importance de la contribution économique et sociale des groupes communautaires au sein du réseau de la santé et des services sociaux au Québec. On y élabore également une stratégie de lutte à la pauvreté basée sur la consolidation de ces organismes voués au mieux-être de la communauté.

Cette stratégie, bien qu'elle s'insère au cœur du débat susmentionné, $n$ 'aborde pas volontairement les questions théoriques qui y sont sousjacentes. Ce texte traduit avant tout l'ébauche d'un projet d'intervention économique et sociale. Aussi certaines hypothèses formulées relèvent-elles essentiellement de l'opinion personnelle des auteurs. 


\section{INTRODUCTION}

Le présent document explore l'importance du réseau des organismes communautaires comme un des éléments importants du secteur émergent de l'économie sociale. Ce secteur peut en effet créer des emplois pour des personnes qui risqueraient autrement d'être exclues du marché du travail tout en permettant de répondre à des besoins essentiels pour la qualité de vie des personnes, besoins auxquels le réseau des services publics n'est plus en mesure de répondre.

Ce texte propose donc de consolider la présence et de faciliter I'expansion des organismes communautaires au Québec. Pour ce faire, une stratégie novatrice s'annonce viable. Il s'agit en fait de favoriser la croissance des dons à des fins de charité et $d^{\prime}$ orienter une partie de cet accroissement, par le moyen d'incitatifs fiscaux, vers le développement du secteur communautaire. Ces dons seraient recueillis dans un fonds de développement ayant pour but d'assurer une source de financement propre au secteur communautaire. II sera démontré que, pour les citoyens autant que pour l'État, un tel investissement dans le domaine de l'économie sociale pourrait $s^{\prime}$ autofinancer complètement à partir des retombées et des effets de leviers qui seraient générés par la mise en œuvre d'une telle stratégie.

Il est aussi proposé que les postes actuellement comblés par des prestataires de l'aide sociale au sein des organismes communautaires puissent éventuellement devenir plus réguliers, que l'allocation supplémentaire de participation reçue par le bénéficiaire lui soit versée par l'organisme et que ce dernier lui accorde, à même ses fonds, un supplément d'allocation. Il y a d'autre part des personnes sans emploi pour lesquelles un travail dans le secteur communautaire peut fournir un emploi valorisant et un retour à l'autonomie financière. Bref, il s'agit d'une stratégie de lutte à la pauvreté qui veut permettre aux personnes d'éviter l'exclusion sociale et de participer à la vie de la société.

\section{L'IMPORTANCE DU SECTEUR COMMUNAUTAIRE}

On compterait environ 90000 organismes sans but lucratif (OSBL) au Québec. De ceux-ci, 54000 sont incorporés et 14000 détiennent un numéro de charité permettant l'émission de reçus de charité (Verret, 1993). Les OSBL sont présents dans des secteurs aussi diversifiés que la protection de l'environnement, la protection du consommateur, l'éducation populaire. Ils sont également actifs au sein de domaines davantage traditionnels comme les organismes de charité d'origine 
religieuse, les fondations, les organismes culturels, les organismes de loisirs. Dans le domaine sociosanitaire, on dénombre plus de 2300 de ces organismes sans but lucratif (ministère de la Santé et des Services sociaux [MSSS], $1995: 5$ ).

\section{Les effectifs des organismes du secteur sociosanitaire}

Le secteur de la santé et des services sociaux comptait, en 1992 1993, quelque 2374 organismes communautaires jugés admissibles ${ }^{1}$ aux subventions du Service de soutien aux organismes communautaires $^{2}$ (MSSS, 1995: 5-6) du ministère de la Santé et des Services sociaux. Ces organismes ${ }^{3}$ (Service de soutien aux organismes communautaires, 1994: 7) avaient à leur emploi 10107 employés réguliers et 14871 employés occasionnels (voir tableau 1). Parmi ces derniers se retrouvaient les employés engagés par l'intermédiaire de certains programmes de développement de l'employabilité du ministère de la Sécurité du revenu (MSR), dont la mesure expérience de travail ${ }^{4}$ (MSR, 1995: 217). Les heures de travail comptabilisées par ces organismes représentent entre 8354 et 9810 travailleurs équivalents temps plein (ETP) selon le critère qu'on utilise ${ }^{5}$. Par comparaison, l'ensemble du réseau des CLSC $^{6}$ compte 16000 salariés, soit l'équivalent de 11000 emplois ETP.

Les organismes communautaires mobilisent également un grand nombre de bénévoles. On parle ici de 339537 personnes qui effectueraient chacune soixante heures de bénévolat par année, en moyenne, dans ces organismes ${ }^{7}$. Cela représente l'équivalent de 11246 ou 13205 emplois ETP si les heures de travail fournies par ces bénévoles avaient été effectuées par des travailleurs salariés.

En somme, travailleurs et bénévoles des organismes communautaires du secteur de la santé et des services sociaux représentent quelque 375000 personnes, soit environ $7 \%$ de la population adulte du Québec.

\section{Les sources de revenu des organismes sans but lucratif}

Les organismes sans but lucratif du secteur sociosanitaire ont généré, en 1992-1993, des revenus globaux de 275 millions $^{8}$ de dollars (voir tableau 1). De ce montant, 70,5 millions provenaient du Service de soutien aux organismes communautaires (SSOC). D'autres instances du gouvernement du Québec, notamment le ministère de la Sécurité du revenu et le ministère de l'Éducation, leur ont versé 39,5 millions. Ils ont aussi puisé 40 millions auprès du gouvernement fédéral. Les organismes ont également généré 125 millions de sources autres que 
gouvernementales, soit par des activités d'autofinancement (74 millions), en subventions de Centraide (38 millions), des municipalités ( 5 millions) et en frais de services ( 8 millions).

Ainsi, l'argent public ne finance pas entièrement les activités de ces organismes. En prenant en considération l'argent qui vient de toutes les sources publiques ( 150 millions), chaque dollar public en génère $0,83 \$ \mathrm{~d}^{\prime}$ autres sources (125 millions). Il se crée donc une sorte d'effet de levier. Si l'on veut accroître l'autonomie financière des groupes communautaires et leur indépendance par rapport au financement gouvernemental, c'est du côté de ces "autres sources» qu'il faut probablement regarder. Cela peut se faire en développant des incitatifs fiscaux destinés à accroître les dons privés à certaines fins, comme nous le verrons plus loin.

\section{Les dépenses des organismes communautaires du réseau}

Les données fournies par les organismes ${ }^{9}$ indiquent des dépenses totales de 241 millions de dollars dont 145 millions, soit $60 \%$, sont consacrés aux salaires (voir tableau 1). Comme on dispose du nombre $d$ 'heures effectuées par le personnel régulier et occasionnel, il est donc possible d'évaluer les taux horaires. Ceux-ci varient de $7,57 \$$ I'heure pour le secteur des organismes de promotion et de services à 13,44 \$I'heure pour le secteur des organismes d'aide aux femmes en difficulté, les deux autres secteurs se situant entre les deux, pour un salaire moyen de 9,54 \$I'heure.

Considérant le nombre d'heures travaillées en moyenne par les employés réguliers, cela représente des revenus annuels moyens respectifs de $9152 \$$ (promotion et services), 14487 (femmes en difficulté), 11288 (jeunes) et $3835 \$$ (maintien à domicile) selon chacun des secteurs ${ }^{10}$. La moyenne générale des revenus annuels des employés réguliers de tous les secteurs se situe ainsi à $7953 \${ }^{11}$.

\section{Évaluation des retombées directes et indirectes}

L'ensemble des dépenses des organismes communautaires œuvrant dans le domaine sociosanitaire génèrent aussi des revenus pour l'État. Ainsi, au seul chapitre de la masse salariale, les 145 millions de dollars dépensés en rémunération génèrent des revenus d'environ 23,4 millions au seul gouvernement du Québec ${ }^{12}$. Cela représente $21,3 \%$ des 110 millions qui sont distribués directement au total par I'État québécois à ces organismes ${ }^{13}$. L'économie serait évidemment plus grande en additionnant les revenus du gouvernement fédéral. 


\section{TABLEAU 1}

Statistiques relatives aux organismes communautaires du secteur des services de santé et des services sociaux, 1992-1993

a) Effectifs

- Nombre d'organismes

- Nombre d'employés réguliers (nombre moyen $d^{\prime}$ heures / année)

- Nombre d'employés occasionnels (nombre moyen d'heures / année)

- Nombre de bénévoles (nombre moyen $d^{\prime}$ heures / année)

\section{b) Revenus}

- Service de soutien aux O.C.

70,5 millions

- Gouvernement du Québec (autres)

39,5 millions

- Gouvernement fédéral

40,0 millions

- Autres sources

125,0 millions

Total $\quad 275,0$ millions

\section{c) Dépenses}

- Salaires

- Autres dépenses

145,0 millions

96,0 millions

Total 241,0 millions

Source: Données obtenues du Service de soutien aux organismes communautaires (SSOC).

Les autres dépenses de fonctionnement des organismes entraînent également des retombées en taxes de vente et en revenus indirects pour les gouvernements. $C^{\prime}$ est aussi un aspect de la question dont il faut tenir compte. Il est possible d'évaluer ces retombées à I'aide des modèles économétriques (Lamonde et al., 1994). On recourt fréquemment à cet égard au modèle interindustriel du Bureau de la statistique du Québec (BSQ). Ce modèle permet d'évaluer les effets indirects qui résultent de l'achat de biens et services par les organismes, de même que les effets indirects de la hausse de consommation des ménages. Si l'on prend comme point de comparaison le secteur de l'industrie des services à la production, les salaires versés par les organismes communautaires et les autres dépenses de production auraient créé environ 2092 emplois additionnels. En retour, ces emplois auraient engendré une masse salariale de 52,3 millions, laquelle aurait à son tour entraîné des rentrées de 8,4 millions pour le gouvernement du Québec. 
Au total, les organismes communautaires du secteur sociosanitaire auraient donc généré des rentrées fiscales de l'ordre de 31,8 millions $^{14}$ en 1992-1993. Ces données ne tiennent cependant pas compte des retombées pour le gouvernement fédéral qu'on peut évaluer à environ 25 millions.

\section{Importance accrue dans un contexte difficile}

En somme, les organismes sans but lucratif (OSBL) sont devenus des acteurs importants au sein du réseau des services de santé et des services sociaux. Cela prend un sens nouveau dans le contexte des limites imposées à la capacité de dépenser de l'État et à la remise en question de plusieurs aspects de l'intervention de celui-ci. Les organismes communautaires du réseau rendent des services personnalisés précieux aux personnes, services que le réseau public ne pourrait plus souvent offrir financièrement. Ces organismes s'ajustent aussi plus rapidement aux nouveaux besoins qui émergent au sein de la population. Il est évident que les ressources mises en place par ces organismes absorbent une partie de la pression de la demande de services qui, autrement, s'exprimerait vers les services professionnels et institutionnels publics.

\section{LA CRÉATION D'UN FONDS DE DÉVELOPPEMENT COMMUNAUTAIRE}

Le secteur des organismes sans but lucratif fait aussi partie du domaine de "l'économie sociale ${ }^{15}$ " (Lévesque et Malo, 1992: 386-387) au sens large. Pourtant, en matière de financement des activités des organismes communautaires, on a jusqu'à maintenant surtout prêté attention aux services directs rendus par ces organismes. Dans certains cas, en particulier celui des subventions distribuées par Centraide, on a d'abord financé les services directs à la population au détriment des dépenses nécessaires de structures, entre autres celles relatives à la masse salariale. Cette lacune a posé des problèmes chroniques de développement et de consolidation aux organismes. On n'a pas suffisamment prêté attention, à cet égard, à l'évaluation des retombées économiques de ces dépenses, notamment en matière de création d'emplois.

Dans un contexte où les interventions de l'État sont remises en cause, il devient pertinent de définir des façons nouvelles d'assurer le maintien et la survie des organismes dispensant des services aux 
personnes nécessiteuses. En ce sens, il appert que la création d'un fonds de développement communautaire constitue une solution novatrice qu'il convient d'explorer.

\section{L'utilité d'un fonds de développement communautaire}

Un relevé rapide permet de dénombrer 213 fonds de développement économique local au Québec (Lévesque et al., 1994). Ces fonds fournissent le capital de risque dont les entreprises ont besoin pour se lancer et se maintenir en affaires.

Par contre, on ne trouve aucun fonds équivalent sur le plan sociocommunautaire. Les fonds à caractère économique sont d'ailleurs parfois nombreux à intervenir sur un même projet en complément l'un de l'autre. On retrouve déjà en bonne partie la même complexité sur le plan social dans la mesure où les organismes communautaires doivent assurer leur financement en puisant dans plusieurs programmes différents. À cette différence qu'il n'y a pas, sur le plan sociocommunautaire, de fonds de développement proprement dit qui permette l'expansion et la consolidation des organismes.

La mission d'un fonds de développement communautaire serait de favoriser la mise sur pied et la consolidation d'organismes actifs dans certains secteurs où existent des besoins sociosanitaires non satisfaits. Il y aurait donc un double objectif, soit de répondre à un besoin réel sur le plan des services requis, mais également de favoriser la création d'emplois réguliers dans ce secteur. Le Québec se doterait ainsi d'un moyen original afin de rattraper le retard qu'il accuse par rapport aux autres provinces et aux États-Unis en matière de développement du secteur communautaire, secteur de l'économie sociale.

\section{Sources de financement possibles}

Au nombre des avenues permettant de financer un tel fonds de développement communautaire, la contribution personnelle des citoyens par le moyen de dons charitables s'avère tout autant souhaitable que réalisable.

Les principales données en ce qui concerne les dons de charité au Québec sont impressionnantes (Verret, 1993). On y constate aussi que les "dons officiels avec reçus » ne représentent qu'une partie de la réalité, puisque bien des gens donnent spontanément pour certaines causes sans exiger un reçu aux fins d'impôt en retour. Par ailleurs, la portion des dons qui est redistribuée en subventions par Centraide ne représente que $11 \%$ de l'ensemble des dons charitables recueillis 
au Québec. Mais cette réalité est appelée à évoluer rapidement au cours des prochaines décennies. On peut en effet s'attendre à ce que les Québécois, qui accusent un retard en regard du reste du Canada, continuent à donner davantage (ibid.).

Ainsi, malgré leur grande générosité, les Québécois se situent effectivement sous la moyenne canadienne en matière de charité privée. En 1989, le don moyen annuel des Québécois (306 \$) représente moins de la moitié $(632 \$)$ du don moyen des résidants des autres provinces. La différence de revenu moyen entre ces deux groupes n'expliquerait qu'une partie de l'écart. On peut plutôt supposer que ce type de financement est moins développé au Québec, parce que l'État occupe une plus grande place dans la prestation des services depuis la révolution tranquille.

Le secteur de la charité privée a cependant connu une croissance remarquable au cours des dernières années au Québec. Si ces tendances se poursuivent, le montant total des dons de toute provenance (particuliers, corporations, fondations, etc.) pourrait atteindre 2,5 milliards de dollars par année en l'an 2000. Pour la seule période de 1993 à 2000, le montant total pourrait s'élever à 13 milliards. Ces chiffres qui semblent excessifs à première vue ne doivent pourtant pas être loin de la réalité lorsqu'on considère les dons effectués en 1989 et le rattrapage que le Québec est appelé à faire à cet égard. On évalue en effet qu'un peu plus de $20 \%$ des adultes québécois font des dons de charité, alors que cette proportion frôle les $50 \%$ en France $^{16}$ (Le Monde, 1995 : 13).

\section{Des mesures incitatives}

L'ampleur d'une telle évolution en matière de dons de charité nous amène à nous interroger sur l'orientation que doit emprunter l'utilisation de cet argent. En particulier, la question se pose à savoir dans quelle mesure cette évolution pourrait être infléchie dans le but de favoriser le développement de l'économie sociale au Québec. Afin $\mathrm{d}^{\prime}$ orienter dans le sens désiré une partie plus importante des dons charitables, on peut envisager à cet égard certaines mesures d'incitation fiscale, notamment les deux hypothèses qui suivent.

Premièrement, il y aurait lieu de valoriser, sur le plan fiscal, les dons de charité accordés à certaines fins précises, dont le développement des services communautaires. La valorisation fiscale est déjà un moyen utilisé aux États-Unis (Business Week, 1995: 65-66): un don de $100 \$$ par exemple donne droit à une déduction de $110 \$$ à 120 \$ sur le rapport d'impôt du contribuable. 
En deuxième lieu, on sait que les personnes qui sont les plus généreuses en dons de charité ${ }^{17}$ sont aussi celles qui investissent le plus dans un régime enregistré d'épargne retraite (REER). Lorsqu'elles atteignent le maximum de contribution, un don de charité pourrait leur donner droit à une cotisation supplémentaire d'un montant égal dans leur REER. Cette cotisation serait également réservée à certaines fins jugées socialement plus désirables. Sachant cependant qu'une bonne partie des déductions possibles pour cotisation dans un REER n'est pas utilisée actuellement, cela réduirait quelque peu l'efficacité $d^{\prime}$ 'une telle mesure.

Par contre, il est prévu qu'il y aura un accroissement rapide des montants de capital transmis par héritage au cours des deux prochaines décennies. Même si l'impôt sur les successions est maintenant aboli, les gains de capitaux transmis par héritage demeurent assujettis à l'impôt. II y a là un potentiel énorme à exploiter éventuellement sous forme de "dons de charité planifiés". Selon cette formule, le capital retourne, exempt d'impôt, aux héritiers après une certaine période, alors que l'usufruit a bénéficié entre-temps à une fondation charitable. Cette source de dons de charité, plus largement connue en milieu anglophone, est encore relativement peu utilisée au Québec.

On se devrait cependant d'ajuster ces hypothèses, afin d'éviter un cumul exagéré $d^{\prime}$ avantages fiscaux, situation qui se produirait si la déduction pour don de charité était valorisée selon la première hypothèse en même temps que le don ouvrait la porte à une déduction supplémentaire pour investissement additionnel dans un REER.

\section{Un développement qui s'autofinance}

Il est permis de croire que les investissements de fonds publics dans le développement du secteur de l'économie sociale pourraient s'autofinancer dans une bonne mesure. Ainsi, un don de $100 \$$ versé par un contribuable à un fonds de développement communautaire lui donnant droit à une déduction fiscale de $30 \%{ }^{18}$ coûterait donc $30 \$$ à l'État québécois. À son tour, ce don de $100 \$$ entraînerait des rentrées supplémentaires de $104 \$$ en raison de la capacité démontrée des organismes communautaires de générer d'autres revenus.

De ce montant total de $204 \$, 122$ \$ en moyenne seraient redistribués en salaires. Cela occasionnerait des rentrées fiscales de 19,70 \$ pour le gouvernement du Québec. Les autres 82 \$ couvrant les diverses dépenses de fonctionnement généreraient pour leur part des revenus additionnels pour l'État (taxes de vente, etc.) qu'on peut évaluer par hypothèse à $5,74 \$$. 
En fait, les $30 \$$ de fonds publics investis au départ sous forme de crédit fiscal auraient entraîné un retour financier direct de 25,44 \$ pour le gouvernement du Québec. Il lui en aurait donc coûté 4,56 \$ pour générer 204 \$ en activités économiques de nature sociale. En poussant davantage l'analyse, on peut estimer que ce coût pour le gouvernement québécois pourrait d'ailleurs être complètement absorbé par les revenus qu'il retirerait des retombées indirectes de ces dépenses. Selon le modèle interindustriel du Québec (Lamonde et al., 1994), les dépenses indirectes qui auraient découlé de ces investissements dans le développement communautaire auraient à leur tour occasionné des revenus de 7,14 \$ à l'État. La dépense aurait donc été rentable pour le gouvernement du Québec pris seul.

Ce calcul ne tient pas compte, par ailleurs, des économies réalisées par la réduction probable de la demande de services professionnels et institutionnels dans le réseau des services de santé et des services sociaux, ni celles occasionnées dans le secteur de la sécurité du revenu par l'embauche possible de bénéficiaires de I'aide sociale.

\section{Des véhicules pour gérer le fonds de développement}

Le véhicule le plus connu actuellement pour amasser de telles sommes et exercer un certain contrôle sur un tel fonds de développement est celui constitué des 17 Centraide répartis dans les différentes régions du Québec. Déjà, les activités subventionnées par les Centraide correspondent au type d'activités dont on veut encourager l'essor dans une perspective de développement de l'économie sociale.

Sur le plan local, certains organismes pourraient également être accrédités afin d'exploiter un fonds de développement communautaire. Il pourrait s'agir notamment des Corporations de développement communautaire $(C D C)$ où siègent déjà des représentants des organismes communautaires du milieu; des Corporations de développement économique communautaire (CDEC) où, en plus des organismes communautaires, sont représentés les autres agents de développement du milieu; des Sociétés d'aide au développement des collectivités (SADC).

Un tel mandat pourrait demander certaines modifications à la mission originale de ces organismes. Ceux-ci, en effet, cherchent d'abord à favoriser le développement "économique» local, mais ces préoccupations ne sont finalement pas si éloignées de celles du développement communautaire local lui-même. 


\section{Des effets de levier}

La formule de fonds de développement communautaire, telle que proposée, repose sur la recherche d'effets de levier. Ces leviers résident notamment dans I'utilisation des dépenses publiques sous forme de remboursements ou de crédits d'impôt, plutôt que sous forme de subventions directes, afin d'en multiplier l'effet.

Aussi, pour les organismes communautaires, on peut escompter que leurs revenus accrus par l'intermédaire d'un fonds de développement sauront susciter à leur tour des rentrées d'argent additionnelles sous d'autres formes, tel l'autofinancement. Enfin, l'accroissement de ces revenus pour les organismes entraîne nécessairement des retombées supplémentaires pour la communauté lorsqu'elles se traduisent en dépenses de fonctionnement ou de consommation.

En somme, les effets de levier engendrés par les investissements "sociaux " créent une dynamique d'entraînement auprès des groupes, des communautés, de la société. Le fait d'utiliser la formule du don de charité, ou une forme assimilée, pour lancer cette dynamique s'inscrit dans une tradition fortement implantée dans notre culture. Tradition qui n'est pas encore arrivée à saturation, lorsqu'on se compare à d'autres (Le Monde, 1995: 13), et qui est vraisemblablement appelée à connaître une expansion importante (Verret, 1993) au cours des prochaines décennies, ne serait-ce qu'en raison du vieillissement de la population et du fait que les personnes âgées sont aussi les plus généreuses en matière de dons charitables.

L'approche proposée vise donc à orienter cette expansion de la charité privée, en partie, dans une direction qui est doublement désirable sur le plan social. Ainsi, tout en contribuant à la consolidation et au développement des organismes communautaires, cette approche trouve sa justification, d'une part, par les activités de services et de solidarité sociale qu'elle engendre et, d'autre part, par les emplois qu'elle procure à des personnes exclues du marché du travail.

\section{LES RETOMBÉES ANTICIPÉES DE LA STRATÉGIE PROPOSÉE}

La mission d'un fonds de développement communautaire, nous l'avons vu, comporte deux aspects fondamentaux, à savoir de permettre aux organismes subventionnés de disposer des ressources nécessaires pour fournir certains services essentiels de nature sociosanitaire que le secteur public ne peut plus entièrement assurer à la population, 
de même que de contribuer à la création d'emplois nouveaux dans le domaine de l'économie sociale. Dans chacun des cas, il est possible de déceler certains impacts qui découlent de leur mise en œuvre.

Les paragraphes qui suivent tentent d'illustrer comment une stratégie de développement fondée, au départ, sur la contribution financière volontaire des particuliers par le moyen de dons charitables peut engendrer des retombées qui bénéficient, à court terme, à certains groupes de la population et, ultérieurement, à la société dans son ensemble.

\section{Des effets préventifs auprès de la population}

Les retombées en matière de services rendus à la population par les organismes communautaires du réseau de la santé et des services sociaux ne sont plus à démontrer ${ }^{19}$ (Hamel, 1995: 966). On connaît de mieux en mieux l'importance des réseaux de soutien naturels et communautaires pour aider les personnes à mieux vivre. Ces réseaux et ces groupes d'entraide ont un effet de prévention et de promotion de la santé et du bien-être auprès des personnes, et cela même si le plus souvent ils ne se conçoivent pas eux-mêmes comme agissant dans ces secteurs (O'Neill et Cardinal, 1994).

Aussi, il semble évident que les ressources mises en place par les organismes communautaires absorbent une partie de la pression de la demande de services qui autrement s'exprimerait vers les services professionnels et institutionnels publics. D'ailleurs, ces organismes s'ajustent plus rapidement aux nouveaux besoins qui émergent au sein de la population. Cependant, force est de constater que l'efficacité et la «rentabilité sociale» des activités effectuées par les organismes communautaires demeurent des réalités encore mal documentées par la recherche sociale au Québec (Bélanger, 1995).

\section{La création d'emplois véritables}

On sait, par ailleurs, que les besoins sociaux en matière de services et d'entraide qui étaient jusqu'à maintenant le plus souvent satisfaits par la famille ou les proches, par les femmes principalement (Fortin, 1995: 951), deviennent plus difficiles à combler notamment en raison de la réduction de la taille des familles, de la généralisation du travail des femmes et du vieillissement de la population.

Aussi, pour plusieurs organismes communautaires, le recours aux bénéficiaires de certains programmes de la sécurité du revenu constitue un instrument essentiel afin de mieux desservir les personnes 
dans le besoin. On peut même poser I'hypothèse que l'apport de ces bénéficiaires est indispensable pour les organismes qui comptent sur un grand nombre de bénévoles et qui fournissent des services directs à la population.

En ce sens, les données révèlent que, en 1992-1993, 1587 projets "expérience de travail » (voir note 4) ont été autorisés dans le réseau de la santé et des services sociaux ${ }^{20}$. Ces projets, représentant 9409 postes différents, ont accueilli 17805 personnes en cours d'année. Ils ont été principalement présentés par des organismes communautaires ( $89 \%$ ) et dans une moindre mesure par des établissements du réseau.

Au total, ce sont $60 \%$ des organismes du secteur sociosanitaire qui font appel, par le programme "EXTRA», à des prestataires de I'aide sociale pour combler certaines tâches ${ }^{21}$. 11 est permis de croire que le travail effectué par ces bénéficiaires de l'aide sociale pourrait tout aussi bien s'accomplir dans le cadre d'un emploi plus traditionnel.

\section{Le potentiel d'emplois dans le secteur sociosanitaire}

Il s'avère difficile de déterminer le nombre exact d'emplois que pourrait engendrer la mise en œuvre d'un fonds de développement communautaire. À titre d'exemple, on peut cependant évaluer qu'un ajout net de 10 millions de dollars en dons de charité, lequel entraînerait des revenus supplémentaires d'une autre dizaine de millions pour les organismes, pourrait se traduire en la création directe d'environ 1000 emplois réguliers et de 1400 emplois occasionnels au sein du secteur communautaire. Lorsqu'on ajoute les 200 emplois indirects qui résulteraient de cet investissement, ce sont donc 2600 personnes de plus qui seraient actives sur le marché du travail.

En matière de création d'emplois, un potentiel énorme existe dans le domaine sociosanitaire. En fait, quelque 9000 nouveaux emplois seraient créés dans la mesure où les organismes communautaires obtiendraient les ressources nécessaires pour consolider les postes actuellement comblés par des prestataires de la sécurité du revenu.

Il y aurait donc intérêt à trouver une façon de consolider une partie de ces emplois que génèrent les organismes communautaires. Il peut en découler des économies importantes pour l'État québécois sous forme de prestations d'aide sociale qu'il n'aurait plus à verser à une partie des bénéficiaires. À cet égard, l'apport de certaines modifications à l'actuel programme expérience de travail (EXTRA, 
MSR, 1995) du ministère de la Sécurité du revenu ${ }^{22}$ permettrait $d^{\prime}$ atteindre un tel objectif.

\section{D'un statut de prestataire à celui de salarié}

La création par le gouvernement fédéral du programme de Transfert social canadien (TSC), qui réunit sous un même chapeau la contribution financière fédérale au titre des programmes établis comme l'éducation postsecondaire, les services de santé et l'ancien régime $d^{\prime}$ assistance publique du Canada ${ }^{23}$ (Gouvernement du Canada, 1994), sera accompagnée d'une importante diminution dans les paiements de transfert vers les provinces. Mais, en même temps, plusieurs barrières seront levées. Une de celles-ci concerne la possibilité, désormais laissée aux provinces, de transformer les prestations d'aide sociale en subventions salariales.

Cette procédure peut se révéler un instrument de développement particulièrement utile en matière de création de nouveaux emplois dans le secteur de l'économie sociale. Elle permettrait notamment $d^{\prime}$ accorder directement les fonds à un organisme communautaire, lequel verserait ensuite un "véritable» salaire à I'ex-prestataire.

La réforme du régime de l'aide sociale qui devrait suivre la parution du rapport du comité Bouchard-Fortin ${ }^{24}$ devrait aussi indiquer les voies susceptibles de maintenir l'incitation au travail chez ces "nouveaux» salariés en évitant dans la mesure du possible de créer des situations où ils n'auraient pas intérêt financièrement à quitter l'aide sociale à cause des avantages collatéraux qu'ils perdraient.

\section{Une occupation valorisante}

La mise en œuvre d'une formule nouvelle comme nous le proposons $\mathrm{n}^{\prime}$ a pas pour effet de favoriser l'émergence d'un ghetto d'emploi ou de cheap labor ou, si l'on veut, de travail ignoble. II y a ici encore deux aspects à considérer. On peut supposer en effet qu'une partie des prestataires trouveront dans ce type d'occupation une forme de participation valorisante et utile à la vie de la société. Cela pourrait être vrai par exemple pour des personnes qui éprouvent des difficultés à réintégrer le marché du travail (Lapierre et Beaulieu, 1995) à cause, notamment, $d^{\prime}$ un niveau de scolarité trop bas, d'un certain âge, pour des jeunes chômeurs possédant peu d'expérience de travail, pour des gens dont les capacités sont limitées. Un grand nombre de ces 
personnes ont peu de chances d'intégrer ou de réintégrer un marché privé de l'emploi qui est de plus en plus compétitif et exigeant.

L'autre aspect concerne la vocation sociale de l'organisme dispensateur de services. On ne peut parler de cheap labor ou de travail ignoble de la même façon quand il s'agit de tâches essentielles au plan humain, mais dont le coût ne pourrait être couvert entièrement par les services publics. Par ailleurs, du côté de l'employé, le choix d'adhérer ou non à une telle mesure devrait être davantage volontaire et la durée de l'engagement pas nécessairement limitée dans le temps (Sylvestre, 1995) de façon aussi stricte qu'elle ne l'est actuellement ${ }^{25}$ (MSR, $1995: 217$ ).

\section{Des emplois non étiquetés}

Bien que I'un des objectifs poursuivis par la mise en place de fonds de développement communautaire soit de faciliter l'accès à un emploi à des personnes souvent laissées en marge du marché du travail, on retiendra qu'il n'est nullement question ici de créer des emplois principalement ou exclusivement réservés à des bénéficiaires de l'aide sociale. Cet aspect doit être pris en considération parmi $d^{\prime}$ autres dans la perspective où cela fait partie des retombées sociales désirables qui sont recherchées.

En ne visant pas uniquement l'embauche de prestataires de la sécurité du revenu, on évite le risque d'étiquetage trop souvent relié à ce type de projets ainsi que le stigmate qui accompagne l'appellation " assisté social ». Cela permet aussi de tenir compte de la nature de chaque projet, certains pouvant demander une main-d'œuvre plus qualifiée. Dans d'autres cas, le dynamisme et la bonne marche du projet peuvent dépendre de la présence de leaders ou d'entrepreneurs sociaux qui ne sont pas nécessairement prestataires de l'aide sociale. On évite également un effet pervers possible où les personnes devraient $d^{\prime}$ 'abord se qualifier à l'aide sociale avant d'avoir accès à un emploi qui les intéresse. Chaque cas doit donc être apprécié au mérite, le nombre de bénéficiaires de l'aide sociale engagés pouvant être l'une des retombées positives du projet présenté.

\section{L'incidence sur l'action bénévole}

La mise en œuvre des activités de tels fonds de développement communautaire pourrait-elle avoir des effets négatifs sur la participation des bénévoles? La question est légitime. II existe souvent des tensions au sein des organismes entre les permanents et les bénévoles. Mais 
il appartient à chaque organisme de trouver l'équilibre requis en fonction de sa dynamique propre. De façon générale, cependant, l'accroissement du nombre de permanents salariés n'a pas nécessairement pour effet de réduire la participation des bénévoles. C'est le contraire qui se passe dans plusieurs organismes où le bénévolat n'a pu se développer qu'avec le support d'une infrastructure stable. Ailleurs, c'est le nombre élevé de bénévoles qui justifie l'engagement de permanents. Par exemple, les centres de bénévolat $n^{\prime}$ auraient certes pas le même rayonnement sans le soutien de leur permanence. Mais le soutien et le développement du bénévolat peuvent aussi être des objectifs assignés aux fonds de développement communautaire.

\section{CONCLUSION}

On le conçoit, les organismes communautaires sont appelés à occuper une place de plus en plus grande au sein du réseau de la santé et des services sociaux. Ils constituent un instrument efficace de mobilisation et de «solidarisation» de la population devant les contraintes imposées par les mutations rapides de notre société.

Ces organismes représentent également un réservoir "d'entrepreneurship social» important et dynamique. Près de 400000 Québécoises et Québécois participent aux diverses activités de services et d'entraide mises en œuvre par le secteur communautaire. Il importe, dans le contexte actuel, de soutenir de telles initiatives. À cet égard, il est permis de croire qu'un fonds de développement communautaire contribuerait, d'une part, à stabiliser les fonds de roulement des organismes et, d'autre part, à favoriser l'essor de nouveaux organismes voués eux aussi au mieux-être de la communauté.

L'importance des réseaux de soutien naturels et communautaires en matière de prévention, de promotion de la santé, de prestation de soins curatifs et de services tout aussi divers qu'essentiels à certains groupes moins favorisés de la société justifie, à première vue, que I'on investisse dans la consolidation et le développement des organismes du secteur sociocommunautaire.

Cette justification se nourrit également du potentiel de création de nouveaux emplois au sein des organismes, emplois générés par la transformation de certains programmes gouvernementaux d'employabilité.

Nous émettons I'hypothèse que les retombées anticipées, autant en matière de services fournis que d'emplois créés, auront un impact sur la pauvreté. En effet, les personnes les plus susceptibles de bénéficier des services sociosanitaires offerts par les groupes communau- 
taires se retrouvent souvent dans des conditions socio-économiques précaires. En ce sens, l'apport du secteur communautaire ne peut que réduire l'écart entre les conditions de vie des personnes moins privilégiées et celles plus fortunées de notre société. Par ailleurs, le développement de ce secteur de l'économie sociale a également pour objectif de permettre l'intégration en emploi de personnes habituellement exclues du marché du travail. À cet égard, l'accès à un emploi constitue souvent l'étape première conduisant à l'affranchissement de la pauvreté.

Cela pose ultimement la question de la capacité et de la volonté de la population et des autres acteurs de contribuer financièrement au développement de telles activités. Car ce sont eux qui seront éventuellement appelés à fournir, du moins en partie, les investissements de départ sous forme de dons de charité, même s'ils sont déductibles d'impôt, et à contribuer à l'autofinancement des groupes par diverses autres activités.

Le potentiel de développement encore inexploité du secteur de l'économie sociale, que certains appellent de façon un peu cynique "l'industrie de la charité ", laisse supposer qu'il est possible de mettre en œuvre un tel chantier social au Québec. II appartiendra à chaque fonds de développement communautaire d'en faire la preuve par ses réalisations.

\section{Notes}

1. Ces organismes œuvraient tous dans le secteur de la santé et des services sociaux même s'ils n'ont pas nécessairement tous été subventionnés.

2. Le Service de soutien aux organismes communautaires (SSOC) a pour mandat de gérer le budget de subvention du ministère de la Santé et des Services sociaux (MSSS). A cet égard, et de concert avec les Régies régionales de la santé et des services sociaux (RRSSS), il évalue les demandes et attribue les subventions aux organismes communautaires qualifiés.

3. Les organismes œuvrant dans le domaine de la santé et des services sociaux sont classifiés par le Ministère sous quatre secteurs d'activité, soit la promotion et les services à la communauté, les services aux femmes et aux conjoints en difficulté, les services à la jeunesse et les services de maintien à domicile.

4. La mesure "expérience de travail» (EXTRA) vise à apporter un soutien et un encadrement pour aider les prestataires à utiliser leurs capacités dans la réalisation de projets communautaires durables, tout en leur assurant un soutien conseil et financier nécessaire à leur engagement.

5. Les heures de travail comptabilisées par les organismes communautaires se chiffrent 
à 15,2 millions de dollars. Ces heures représentent 8354 ETP si l'on prend une année complète de travail ( 35 heures $\times 52$ semaines $=1820$ heures $/$ année) ou 9810 ETP si l'on prend les 1550 heures / année habituelles du réseau.

6. Centre local de services communautaires. II existe environ 160 CLSC au Québec.

7. Cela représente 20,4 millions d'heures de bénévolat.

8. Les OSBL financés par le Service de soutien aux organismes communautaires (SSOC) joignent à leur demande de subvention des données pertinentes qui permettent de connaître un peu mieux leur réalité et leur importance au sein du réseau. Ces données portent sur l'année 1992-1993 et sont incluses dans la demande de subvention 19931994 de ces organismes. Les demandes ayant été préparées en cours d'année 19931994, plusieurs données notamment financières ne sont pas complètes et reposent sur des extrapolations. Ces extrapolations ne représentent d'ailleurs que $9,3 \%$ du total et n'en changent pas la nature.

9. Les réserves quant à l'absence de certaines données concernant les revenus des organismes s'appliquent également en ce qui concerne leurs dépenses. Ici, aussi, l'absence de ces données nous amène à extrapoler.

10. Le revenu annuel est évalué en fonction des heures rémunérées par les organismes communautaires et ne comprend pas les autres sources de revenu possibles des personnes. Ainsi, 205 organismes apparaissent à la fois dans les secteurs de promotion et services et de maintien à domicile. Dans la mesure où une même personne pourrait recevoir une rémunération sous les deux chapeaux, cela aurait pour effet de sousestimer son revenu global réel.

11. Selon le calcul suivant: moyenne de 833,74 heures travaillées par année multipliée par un taux horaire moyen de 9,54\$ = salaire annuel moyen de $7953 \$$.

12. Le taux moyen d'imposition sur le revenu est d'environ $6,99 \%$, celui des cotisations au régime des rentes du Québec (RRQ) de 1,19\%, à la régie de l'assurance-maladie du Québec (RAMQ) (part de l'employeur) de 3,25\% et celui de la Commission de la santé et de la sécurité du travail (CSST) de 4,69\%, pour un total de 16,12\%.

13. Il s'agit du total de 70,5 millions de dollars du SSOC et des 39,5 millions provenant d'autres instances de l'État.

14. Il s'agit des revenus fiscaux directs de 23,4 millions auxquels s'ajoutent les 8,4 millions de retombées indirectes.

15. "L'expression "économie sociale" est très peu utilisée au Québec. [...] Concrètement, l'expression est utilisée pour désigner moins les coopératives traditionnelles que les nouvelles coopératives et les entreprises associatives qu'on appelle entreprises communautaires et alternatives. Autrement dit, l'expression sert en priorité pour désigner les entreprises qui tentent de concilier impératifs économiques et impératifs sociaux et qui reposent essentiellement sur le dynamisme des collectivités locales et donc sur une participation des citoyens ou encore des travailleurs directement impliqués. "

16. Le phénomène des dons de charité est en croissance rapide en France où la proportion de donateurs est passée de 42,8\% de la population en 1990 à 49,6\% en 1993.

17. À l'exception des personnes qui ont atteint l'âge de la retraite.

18. Le crédit d'impôt pour dons de charité est actuellement de $20 \%$ pour l'État québécois. Il est important de garder en mémoire que tous les raisonnements présentés ici excluent toutes les retombées possibles pour le gouvernement fédéral.

19. L'auteur propose que: " [...] la participation active du milieu communautaire [...] remplit un rôle important dans la gestion de certains services, dans l'amélioration des conditions de vie et dans la réinsertion sociale des plus démunis. Sur le plan de la santé et des services sociaux, le milieu communautaire joue un rôle similaire. »

20. Données obtenues auprès du Service de soutien aux organismes communautaires. 
21. Pour réaliser ces projets, les organismes promoteurs ont reçu un total de 8,14 millions de dollars de la part du ministère de la Sécurité du revenu. Ce montant représente une prime de 100 \$ versée à l'organisme communautaire pour assurer l'encadrement de chaque participant au projet. Les établissements du réseau recevaient encore à cette période $50 \$$ par mois pour assumer les mêmes fonctions.

22. Ce ministère a été aboli en février 1996 lors du premier remaniement ministériel du premier ministre Lucien Bouchard, nouvellement entré en fonction. II a été remplacé par le ministère de l'Emploi et de la Solidarité, dirigé par la ministre Louise Harel.

23. Le régime d'assistance publique du Canada constituait le programme de transfert financier aux provinces par lequel le gouvernement fédéral assumait une partie du financement des services sociaux, notamment en matière d'aide sociale.

24. Le comité Bouchard-Fortin est un comité d'experts, mis sur pied en juin 1995 par madame Jeanne Blackburn, alors ministre de la Sécurité du revenu, ayant pour mandat de proposer un nouveau régime en matière $d^{\prime}$ aide sociale. Le rapport du Comité doit être remis au gouvernement en février 1996 en vue d'être débattu publiquement et faire l'objet d'un projet de loi à la fin de l'année 1996. Au moment de soumettre le présent article pour publication, le comité Bouchard-Fortin n'avait pas encore déposé son rapport.

25. Le programme EXTRA amène les personnes participantes à collaborer à la réalisation de projets communautaires d'une durée habituelle de douze mois, à raison de 80 heures par mois, et peut être prolongé jusqu'à 15 mois. La durée minimale est de six mois.

\section{Références bibliographiques}

BÉLANGER, J.-P. (1995). "L'évaluation et la transformation des systèmes ", communication présentée dans le cadre du colloque: Évaluer pourquoi?, organisé par le CQRS, congrès de l'ACFAS, Chicoutimi, 23 mai 1995.

BUSINESS WEEK (1995). "GOP's blind faith in charity », 6 mars : 65-66.

FORTIN, A. (1995). "La famille, premier et ultime recours", Traité des problèmes sociaux. Québec: IQRC, p. 947-962.

GOUVERNEMENT DU CANADA (1994). La sécurité sociale dans le Canada de demain: document de travail, Développement des ressources humaines Canada, octobre, 102 pages.

HAMEL, P. (1995). "L'action communautaire ", Traité des problèmes sociaux. Québec: IQRC, p. 963-980.

LAMONDE, P. et al. (1994). Impact économique et fiscal des investissements du fonds de solidarité des travailleurs du Québec (FTQ), 1984-1993. Montréal : INRS-Urbanisation, 126 pages.

LAPIERRE, R. et C. BEAULIEU (1995). Relance 1994 auprès des prestataires de la sécurité du revenu ayant participé à un programme de développement de l'employabilité ou d'intégration en emploi. Québec: Direction de la recherche, de l'évaluation et de la statistique, ministère de la Sécurité du revenu, 81 pages. 
LE MONDE (1995). "Les dons et le bénévolat ont fortement progressé », 4 avril : 13.

LÉVESQUE, B. et al. (1994). "Les fonds régionaux et locaux de développement: le poids de l'environnement», communication présentée dans le cadre du colloque des Septièmes entretiens Jacques Cartier: Croissance de l'entreprise en région; financement et accompagnement de projets; stratégies de développement, Lyon.

LÉVESQUE, B. et M.-C. MALO (1992). "L'économie sociale au Québec: une notion méconnue, une réalité économique importante », dans Économie sociale : entre économie capitaliste et économie publique, J. Defourny - J. L. Monzon Campos (dir.), p. 385-446.

Ministère de la SANTÉ ET DES SERVICES sociaux (1995). Programme de soutien aux organismes communautaires 1996-1997, Gouvernement du Québec, 32 pages.

MINISTÈRE DE LA SÉCURITÉ DU REVENU (1995). De l'aide sociale à la sécurité du revenu; rapport statistique 1993-1994, Gouvernement du Québec, 222 pages.

O'NEILL, M. et L. CARDINAL (1994). " Health promotion in Quebec: did it ever catch on?", Health promotion in Canada: provincial, national and international perspectives, A. Pederson, M. O'Neill, I. Rootman et W.B. Saunders (dir.), Toronto, p. 262-283.

SERVICE DE SOUTIEN AUX ORGANISMES COMMUNAUTAIRES (1994). Répertoire des ressources communautaires subventionnées 1993-1994. Québec: ministère de la Santé et des Services sociaux, 166 pages.

SYLVESTRE, C. (1995). Synthèse des résultats des études d'évaluation en matière de développement de l'employabilité et d'intégration en emploi. Québec: Direction de la recherche, de l'évaluation et de la statistique, ministère de la Sécurité du revenu, 63 pages.

VERRET, A. (1993). Profil philanthropique du Québec : qui donne et combien. Québec: Le Centre québécois de philanthropie inc., 112 pages. 